\title{
Manifestações Clínicas e Laboratoriais de Polimiosite em um Caso de Leptospirose
}

\section{Clinical and Laboratorial Features of Polymyositis in a Case of Leptospirosis}

\author{
Carolina de Souza Müller ${ }^{(1)}$, Acir Rachid Filho ${ }^{(2)}$, David Cezar Titton ${ }^{(2)}$, Eduardo Santos Paiva ${ }^{(2)}$
}

\section{RESUMO}

Nem sempre é possível estabelecer uma clara distinção entre as doenças musculares de causa infecciosa e as miosites idiopáticas. Há situações, inclusive, em que se considera existir uma relação causal entre ambas as doenças. Descrevemos o caso de uma paciente com manifestações clínicas e laboratoriais de polimiosite que apresentou infecção precedente de leptospirose, em que se considera a hipótese de ação de "gatilho" do agente infeccioso no quadro muscular inflamatório.

Palavras-chave: polimiosite, leptospirose, rabdomiólise, anti-Jo-1.

\section{INTRODUÇÃO}

Há na literatura inúmeros relatos de doenças infecciosas atuando como "gatilhos" no desencadeamento da doença muscular inflamatória. Entre os trabalhos brasileiros, destaca-se o de Cossermelli e cols. ${ }^{(1)}$, que descreve um quadro de polimiosite na vigência da instalação clínica de doença de Chagas. Reimers et $a^{(2)}$ descreveram quatro casos de miosite causada por Borrelia burgdorferi, sendo a doença de Lyme também a etiologia de doença muscular inflamatória em outro caso mais recente descrito por Horowitz et $\mathrm{al}^{(3)}$. Magid et al ${ }^{(4)}$ relataram a associação de doença muscular inflamatória e infecção na vigência de toxoplasmose aguda. São todos casos de pacientes com miosite idiopática, em que altos títulos de anticorpos contra os agentes infecciosos são detectados, sugerindo que a doença inflamatória possa ser uma conseqüência tardia da infecção por esses agentes.

Relatamos o caso clínico de paciente com polimiosite, que teve o quadro clínico exacerbado pela infecção por lep-

\section{ABSTRACT}

Sometimes, it is not possible to establish an obvious distinction between the muscular manifestations due to infectious diseases and the idiopathic myositis. In fact, in some situations it is believed there is a causal relationship between those diseases. We describe the case of a patient with clinical and laboratorial features of polymyositis who had developed leptospirosis previously. The hypothesis of the infectious agent acting as a "trigger" to the muscular disease is discussed.

Keywords: polymyositis, leptospirosis, rhabdomyolysis, anti-Jo-1.

tospirose. Inúmeras das complicações clínicas desenvolvidas pela paciente foram decorrentes desta associação inusitada, e foram de difícil discriminação, pois ambas enfermidades apresentam aspectos clínicos comuns.

\section{RELATO DE CASO}

Paciente feminina de 19 anos foi internada por quadro de mialgia, astenia, náuseas e vômitos de evolução há três meses. Havia relato de picos febris e uso de amoxicilina sem resolução dos sintomas. À admissão, apresentava força muscular grau II e dores musculares difusas. A incapacidade de deglutição obrigou a instalação de sonda nasogástrica. Não havia, no entanto, evidências de comprometimento da musculatura respiratória. Exames laboratoriais revelavam CK de $135.000 \mathrm{U} / \mathrm{L}$ (normal de 26 até $142 \mathrm{U} / \mathrm{L}$ ), com transaminases e $\mathrm{LDH}$ igualmente elevadas $(\mathrm{SGOT}=2.150$ $\mathrm{U} / \mathrm{L}, \mathrm{SGPT}=907 \mathrm{U} / \mathrm{L}$ e $\mathrm{LDH}=4.357 \mathrm{U} / \mathrm{L}$, para valores normais de até $40 \mathrm{U} / \mathrm{L}$ para ambas as enzimas hepáticas, e

Departamento de Rematologia da Universidade Federal do Paraná (UFPR). Recebido em 22/02/06. Aprovado, após revisão, em 02/10/06.

1. Residente de Reumatologia da UFPR.

2. Professor Adjunto de Reumatologia da UFPR.

Endereço para correspondência: Carolina de Souza Müller, Rua Geraldo Lipka, 65/1301, CEP 81200-590, Curitiba, PR, Brasil, telefone/fax: (41) 3373-6894, e-mail: carolinadesmuller@yahoo.com.br 
de até $190 \mathrm{U} / \mathrm{L}$ para as LDH). O hemograma era normal, bem como os valores de bilirrubina, fosfatase alcalina e o raio X de tórax. A avaliação da função renal revelava insuficiência renal aguda oligúrica, com creatinina $=5,2 \mathrm{mg} / \mathrm{dL}$, hematúria e proteinúria. O potássio era de $5,3 \mathrm{mg} / \mathrm{dL}$ (normal de 3,5 a $5,5 \mathrm{mg} / \mathrm{dL}$ ) e o fósforo $=5,7$ (normal de 2,8 a $4,5 \mathrm{mg} / \mathrm{dL}$ ). Devido à epidemiologia positiva e quadro clínico sugestivo, foi realizada coleta de exame sorológico para leptospirose. No entanto, no momento da admissão e também posteriormente, não se considerou a instituição de terapia antibiótica específica. Procedeu-se à realização de eletroneuromiografia, que foi compatível com padrão miopático, e biópsia muscular (músculo bíceps braquial), que revelou miopatia ativa sugestiva de miopatia isquêmica com achado de alterações predominantemente perifasciculares. Clínica e laboratorialmente estabeleceu-se o diagnóstico de polimiosite, com instituição de metilprednisolona em pulso por três dias $(\mathrm{lg} / \mathrm{dia})$, seguindo-se o uso de prednisona $1 \mathrm{mg} / \mathrm{kg} / \mathrm{dia}$, a que foi posteriormente acrescentado azatioprina na dose de $150 \mathrm{mg} /$ dia. O FAN (anticorpo antinuclear) era de $1 / 40$ anti-citoplasma positivo padrão fibrilar/filamentar e o anti-Jo-1 de $1: 3.200$. $O$ perfil ENA (anti-Ro, anti-La, anti-Sm, anti-RNP) foi negativo. Realizou-se TAC de tórax com achado de infiltrado reticular fino na periferia posterior dos lobos inferiores, sugestivo de pneumonite intersticial. O eletrocardiograma (ECG) e o ecocardiograma eram normais. A investigação quanto possível doença neoplásica associada não revelou alterações. A recuperação do quadro renal foi completa, com hidratação vigorosa e reposição de cálcio. A infecção por leptospira veio a ser confirmada por exame sorológico (IgM ELISA positivo) após 15 dias de internamento, sendo posteriormente ratificada por exame de PCR urinário. Nesta ocasião, visando a possível identificação de componentes da leptospira ou de seus produtos no tecido muscular, foi realizada análise imunohistoquímica do material obtido da biópsia do músculo bíceps-braquial. O resultado, no entanto, foi negativo para a presença de leptospira na amostra analisada. Apesar da melhora significativa apresentada pela paciente seguindo-se o pulso inicial de metilprednisolona, a recuperação posteriormente foi mais lenta, com melhora inicial da força muscular em extremidades, mas manutenção da fraqueza muscular proximal (grau II). Durante o internamento, diagnosticou-se anemia hemolítica com exame de Coombs direto reagente, havendo a necessidade de transfusão de duas unidades de papa de hemácias. Após mais de 60 dias internada, devido à dor na mobilização do quadril, foram realizados exames de imagem do quadril (raio X simples, ultra-sonografia e ressonância magnética) que demonstraram a presença de calcinose em coxas. Por ocasião da alta, completados 80 dias de internamento, a paciente mantinha-se sem possibilidade de deambulação e com força muscular comprometida em musculatura proximal dos membros. A CK na alta era de 799 U/L.

\section{DISCUSSÃO}

Pacientes com doença muscular inflamatória idiopática, nas suas diferentes formas de apresentação, podem desenvolver auto-anticorpos. Auto-anticorpos miosite específicos estão presentes quase que exclusivamente na polimiosite e dermatomiosite ${ }^{(5)}$. Cada um desses auto-anticorpos parece estar associado a características clínicas em comum, a um tipo de HLA predominante, a determinado padrão de apresentação de doença e possivelmente de resposta à terapia ${ }^{(6)}$. No caso relatado, a paciente apresentava altos títulos de anti-Jo-1, o mais prevalente auto-anticorpo presente nas miopatias inflamatórias idiopáticas e caracteristicamente associado à síndrome anti-sintetase ${ }^{(5,7)}$. No entanto, além do comprometimento pulmonar, assintomático, a paciente não apresentava fenômeno de Raynaud, artrite, picos febris ou alterações compatíveis com "mãos de mecânico". O FAN apresentou-se em baixos títulos e com padrão não característico. Este achado associado ao perfil ENA nãoreagente contribuiu para diminuir a possibilidade de miosite associada a outras doenças do tecido conjuntivo ${ }^{(8)}$.

O diagnóstico de leptospirose foi estabelecido apenas mais tardiamente na evolução do quadro, quando os exames solicitados à admissão foram disponibilizados. Apesar do resultado negativo obtido no caso apresentado, mas ressaltando-se a importância de um diagnóstico mais precoce da leptospirose, um estudo brasileiro quanto à análise imunohistoquímica do músculo gastrocnêmio na detecção da leptospira e de seus produtos revelou-se de grande valor. Juntamente ao achado histopatológico de miosite, o método imunohistoquímico possibilitou a identificação da etiologia infecciosa em 94,5\% dos pacientes analisados ${ }^{(9)}$.

Em casos leves de leptospirose é rara a persistência de anticorpos $\operatorname{IgG}$ e $\operatorname{IgM}$ por longos períodos. Finster et $a^{(10)}$ descreveram, entretanto, um caso de leptospirose leve em que, apesar do tratamento efetivo, houve persistência desses anticorpos por três anos.

Tanto na forma anictérica da leptospirose como na síndrome de Weil, mas com maior intensidade nesta última, os achados radiológicos associados às manifestações respiratórias são de lesões compatíveis com pneumonite intersticial 
hemorrágica, com infiltrados localizados ou difusos ${ }^{(11)}$. Du Couedic et al ${ }^{12)}$ demonstraram a presença de hemorragia alveolar em todos os casos analisados de leptospirose com envolvimento pulmonar, e hemorragia alveolar oculta na maioria dos pacientes com leptospirose sem nenhum sintoma respiratório. Portanto, no caso apresentado, o envolvimento pulmonar intersticial em bases, apesar de bastante sugestivo de polimiosite, especialmente na presença de anti-Jo-1, não afasta a etiologia pela leptospira.

$\mathrm{Na}$ síndrome de Weil tardia, Watt et al ${ }^{(13)}$ demonstraram comprometimento das musculaturas estriada e cardíaca, com 37\% dos pacientes apresentando miosite e $39 \%$, anormalidades ao ECG . Ambas as manifestações foram relacionadas à gravidade da leptospirose. No curso da polimiosite, a maioria dos pacientes evolui com períodos de exacerbação e remissão do acometimento muscular, embora possa apresentar doença persistentemente ativa. No caso descrito, a paciente, apesar da terapia instituída, não apresentou a melhora esperada, tanto clínica como laboratorialmente, sugerindo um possível efeito aditivo da lesão muscular pela leptospira e pela polimiosite.

Há relatos na literatura de anemia hemolítica presente tanto na vigência de leptospirose como de polimiosite. $\mathrm{Na}$ leptospirose, estudos apontam a injúria à membrana do eritrócito, decorrente da ação de fosfolipases produzidas pelo agente infeccioso, como possível causa do processo hemolítico ${ }^{(14)}$. Solmazgul et al ${ }^{(15)}$ descreveram caso de síndrome de Weil com achados de anemia hemolítica controlada somente após uso de imunoglobulina intravenosa.

\section{REFERÊNCIAS}

1. Cossermelli W, Friedman H, Pastor EH, et al: Polymyositis in Chagas's disease. Ann Rheum Dis 37: 277-80, 1978.

2. Reimers CD, Pongratz DE, Neubert U, et al: Myositis caused by Borrelia burgdorferi: report of four cases. J Neurol Sci 91: 215-26, 1989

3. Horowitz HW, Sanghera K, Goldberg N, et al: Dermatomyositis associated with Lyme disease: case report and review of Lyme myositis. Clin Infect Dis 18: 166-71, 1994.

4. Magid SK, Kagen LJ: Serologic evidence for acute toxoplasmosis in polymyositis-dermatomyositis. Increased frequency of specific anti- toxoplasmosis IgM antibodies. Am J Med 75: 313-20, 1983.

5. Love LA, Leff RL, Fraser DD, et al: A new approach to the classification of idiopathic inflammatory myopathy: Myositisspecific autoantibodies define useful homogeneous patient groups. Medicine 70: 360-74, 1991.

6. Miller FW, Twitty SA, Biswas T, Plotz PH: Origin and regulation of a disease-specific autoantibody response: antigenic epitopes,
Embora a anemia hemolítica possa fazer parte do quadro clínico de inúmeras doenças auto-imunes, é rara sua associação com polimiosite, embora já descrita na literatura $^{(16)}$. Como a paciente apresentava exame de Coombs reagente, atribuiu-se à anemia hemolítica etiologia mais provável auto-imune, embora não se possa descartar possível contribuição da infecção por leptospira.

$\mathrm{Na}$ leptospirose é comum o comprometimento precoce da função renal, caracterizado por nefrite túbulo-intersticial e atribuído ao efeito tóxico de um componente da membrana externa da leptospira ${ }^{(17)}$. No caso descrito, apesar da ausência de dosagem de mioglobina, acredita-se que a rabdomiólise tenha também contribuído no comprometimento da função renal da paciente, embora, em um estudo envolvendo pacientes com leptospirose, a associação entre mioglobina e insuficiência renal não pôde ser estabelecida ${ }^{(18)}$. Em um trabalho de autores brasileiros, demonstrou-se não haver melhor evolução da leptospirose e insuficiência renal aguda em pacientes tratados com penicilina quando comparados àqueles que não receberam este tratamento ${ }^{(19)}$.

Como a paciente apresentava polimiosite, não se pode desconsiderar a ocorrência, embora rara, de mioglobinúria nas formas graves da doença $a^{(20)}$.

Em resumo, destaca-se a associação inusitada de polimiosite e leptospirose, resultando em doença de curso grave e atípico, com pobre resposta à terapia instituída.

Declaramos a inexistência de conflitos de interesse.

spectrotype stability, and isotype restriction of anti-Jo-1 autoantibodies. J Clin Invest 85: 468-75, 1990.

7. Plotz PH, Rider LG, Targoff IN, et al: Myositis: Immunologic contributions to understanding cause, pathogenesis, and therapy. Ann Intern Med 122: 715-24, 1995

8. Reichlin M, Arnett FC: Multiplicity of antibodies in myositis sera. Arthritis Rheum 27: 1150-6, 1984.

9. Uip DE, Amato Neto V, Duarte MS: The early diagnosis of leptospirosis by demonstrating antigens via an immunohistochemical exam of the hamstring muscle. Rev Inst Med Trop Sao Paulo 34: 375-81, 1992.

10. Finsterer J, Stollberger C, Sehnal G: Mild leptospirosis with three-year persistence of IgG- and IgM- antibodies, initially manifesting as carpal tunnel syndrome. J Infect 5: 67-70, 2005.

11. Veronesi R, Focaccia R: Tratado de Infectologia. São Paulo: Editora Atheneu, 1996.

12. Du Couedic L, Courtin JP, Poubeau P, Tanguy B, Di Francia M, Arvin- Berod C: Patent and occult intra-alveolar hemorrhage in leptospirosis. Rev Mal Respir 15: 61-7, 1998. 
13. Watt G, Padre LP, Tuazon M, Calubaquib C: Skeletal and cardiac muscle involvement in severe, late, leptospirosis. J Infect Dis 162: 266-9, 1990.

14. Trowbridge AA, Green JB 3rd, Bonnett JD, Shohet SB, Ponnappa BD, McCombs WB 3rd: Hemolytic anemia associated with leptospirosis. Morphologic and lipid studies. Am J Clin Pathol 76: 493-8, 1981.

15. Solmazgul E, Turhan V, Unver S, Demirci M, Nalbant S, Danaci M: A case of Weil's syndrome developing steroid resistant immune haemolytic anaemia. Scan J Infect Dis 37: 700-2, 2005.

16. Takahashi C, Sasaki N, Kawabata M, Sakamoto S, Kito S: A case of autoimmune hemolytic anemia complicated with polymyositis.
Rinsho Ketsueki 14: 1033-9, 1973.

17. Yang CW, Wu MS, Pan MJ: Leptospirosis renal disease. Nephrol Dial Transplant 16: 73-7, 2001.

18. Martinelli $\mathrm{R}$, Luna MA, Rocha $\mathrm{H}$ : Is rhabdomyolisis an additional factor in the pathogenesis of acute renal failure in leptospirosis? Rev Inst Med Trop Sao Paulo 36: 111-4, 1994.

19. Daher EF, Nogueira CB: Evaluation of penicillin therapy in patients with leptospirosis and acute renal failure. Rev Inst Med Trop Sao Paulo 42: 327-32, 2000.

20. Kim HW, Choi JR, Jang SJ, Chang YS, Bang YS, Pank CW: Recurrent rhabdomyolysis and myoglobinuric acute renal failure in a patient with polymyositis. Nephrol Dial Transplant 20: 2255-8, 2005. 